\title{
La producción científica de monografías españolas sobre publicidad (1971-2001)
}

\author{
A bibliographical analysis of Spanish academic monographs on advertising
}

\author{
María Jesús MARTínez Pestaña \\ Universidad Complutense de Madrid, Facultad de Ciencias de la Información, C/ Ciudad Universitaria, 28040 Madrid, España \\ maria.j.m.pestana@gmail.com
}

\begin{abstract}
Resumen
Se estudia la producción científica de monografías sobre publicidad en España desde 1971 hasta el año 2001. Los aspectos evaluados han sido: producción anual, producción por autores, producción por autores en colaboración, producción institucional, y producción por idiomas. Para este estudio se han tenido en cuenta tanto las monografías realizadas en el ámbito académico como las procedentes del mundo profesional, dado que las contribuciones de los profesionales de la publicidad son abundantes. Se ha confirmado la consolidación de la publicación de monografías, así como la consolidación de la cooperación entre autores a partir de los años 1990. La creación de licenciaturas de Publicidad en diferentes universidades también ha repercutido en una mayor producción científica desde las universidades. El castellano es la lengua predominante.
\end{abstract}

Palabras clave: Producción científica. Análisis bibliométrico. Monografías. Publicidad. España.

\section{Introducción}

Si bien es verdad que la comunicación científica rápida se hace a través de la publicación de artículos en revistas especializadas, la monografía, en contrapartida, permite, en su extensión, el desarrollo específico de un tema en toda su amplitud. Es más, las modificaciones en los hábitos de los autores e investigadores ha resultado en una mayor abundancia de trabajos en cooperación.

La creación de la Licenciatura de Publicidad (Méndiz Noguero, 2000; Martínez Pestaña, 2004) en la universidad española en el año 1971 generó, sin duda, un gran movimiento productivo en la edición de monografías de autores españoles. Si bien el Instituto Nacional de Publicidad había sido el motor científico de los textos publicitarios, editando y traduciendo hasta esa fecha, a partir de la instauración de los estudios universitarios especializados, la edición de obras monográficas de autoría nacional desde las universidades recibió un gran impulso. Paralelamente, el asentamiento de la actividad

\begin{abstract}
The scientific production of monographs on advertising in Spain is studied from 1971 to 2001 . The analysis that have been carried out are: yearly productivity, productivity by author, co-authorship, institutional productivity, and language of the monograph. Both academic and professional monographs have been taken in account, given that the contribution of professionals from advertising companies is large. It has been proved the consolidation of monographic publications, as well as the cooperation between authors after 1990. The creation of grades in Advertising in different Spanish universities has resulted in increased production from the universities. Spanish is the main language.
\end{abstract}

Keywords: Scientific production. Bibliometric analysis. Spain. Monographs. Advertising.

publicitaria nacional motivó la producción de obra monográfica de sello nacional.

Recordemos que hasta el año 1991 eran tres las universidades que contaban con la Licenciatura de Publicidad: la Universidad Complutense de Madrid, la Universidad Autónoma de BarceIona y la Universidad del País Vasco. A partir de 1991 incorporarían estudios de publicidad, el CEU de San Pablo (Valencia), el Colegio Universitario de Segovia (adscrito a la Universidad Complutense de Madrid), la Universidad Europea de Madrid, la Universidad de Navarra, la Universidad de Málaga, la Universidad Ramón Llull, la Universidad de Vigo, la Universidad Antonio de Nebrija, la Universidad Pontificia de Salamanca, la Universidad Jaume I de Castellón y la Universidad de Alicante.

Es importante destacar que es bastante frecuente que el profesional publicitario participe de la actividad docente universitaria. Esta circunstancia ha favorecido, sin duda, la producción de trabajos de carácter científico y profesional. Destaquemos que la actividad publicita- 
ria es una de las profesiones que en nuestro país goza de más movilidad.

Hay un precedente fundamental en los análisis de producción científica sobre publicidad. En el año 1983 Fernando Martin publicó el primer catálogo bibliográfico sobre trabajos publicitarios. Aunque esta extensa obra incluye trabajos no estrictamente publicitarios resulta de gran importancia, pues es el primer trabajo bibliográfico que incluye un extenso repertorio sobre obras publicitarias, científicas y no científicas. En nuestra opinión es el primer trabajo tendente a establecer la producción científica y no científica sobre publicidad.

\section{Objeto}

El objeto de este trabajo es analizar la producción de monografías de temas publicitarios realizadas en nuestro país durante el período comprendido entre 1971 y 2001. Para el análisis de la producción científica hemos aplicado técnicas bibliométricas de acuerdo con las evaluaciones que deseábamos realizar (Zulueta, 1999, 2002; López Piñero, 1992c, 1992d; López López, 1996b). En primer lugar, hemos analizado la producción global y su evolución durante el período de estudio. En segundo lugar, hemos prestado atención a la autoría de los trabajos y los niveles de mayor productividad de los autores. Asimismo, en tercer lugar, hemos prestado atención a los niveles de cooperación entre autores. En cuarto lugar, se ha analizado la productividad institucional, tanto la de los autores adscritos a universidades como los vinculados a actividades profesionales relacionadas con la comunicación. Finalmente, en quinto lugar, también se han analizado las lenguas en que se han escrito los diferentes trabajos.

\section{Metodología}

Para realizar los diferentes análisis en las diferentes evaluaciones se ha creado una base de datos de tipo relacional para así facilitar el intercambio de datos (Zulueta, 2002, p. 117-119). Los trabajos incluidos son monografías españolas, es decir, trabajos que estudien aspectos específicos de tema publicitario, realizados por uno o más autores españoles o vinculados a instituciones nacionales. Las monografías incluidas plantean hipótesis, aunque no siempre sea muy clara la metodología empleada por el autor, o bien expresan algún problema o aportan información sobre aspectos relativos a la publicidad. Hemos incluido sólo aquellas monografías que cuentan con ISBN, o número de Depósito legal en las monografías anteriores a 1972. En el caso de varias impresiones, hemos considerado la primera; en ediciones revisadas, la última ha sido la elegida.

Inicialmente, consultamos la base de datos del ISBN del Ministerio de Cultura, como también consultamos la de la Facultad de Ciencias de la Información de la Universidad Complutense, por ser ésta la que acogió inicialmente la primera Licenciatura de Publicidad. Dados los errores en la base de datos del ISBN en el primer caso, y lo incompleto de los fondos en el segundo, nos limitamos a la búsqueda en los catálogos de la Biblioteca Nacional de España y a su consulta manual. Para las obras del período más antiguo nuestra fuente de referencia fue la obra de Martín Martín (1983) por ser la más completa, especialmente para el período inicial hasta los años 1980.

Los datos incluidos en la base de datos creada ad hoc cuentan con la información precisa para la realización de las diferentes evaluaciones fijadas de antemano. Se ha insertado en la base de datos la siguiente información para cada monografía: autor o autores de la obra, título completo y subtítulos si los hubiera, año de edición, adscripción institucional del autor en el momento de la publicación de la obra, editor, lugar de edición, y lengua en que se ha publicado la obra. Hemos computado un total de 736 monografías, cuyos datos hemos vertido a la base de datos. A continuación, vamos a proceder a explicar los diferentes análisis cuantitativos realizados.

\section{Evaluación de la producción por años}

Aunque el volumen de 736 monografías corresponde a la producción global para el período de 1971 al año 2001, sin embargo, el volumen muestra diferentes niveles productivos a lo largo de los años (Tabla I). Así, el año 1971 arranca con una producción de 22 monografías. Aunque sólo representa un porcentaje del $2,99 \%$ respecto al conjunto de la producción global, no volveremos a encontrar un volumen similar hasta el año 1977. No cabe duda que el impulso del Instituto Nacional de Publicidad fue un gran motor en los años anteriores a 1971. De hecho, en los años posteriores a éste, las cifras serán sustancialmente menores. Para los años 1972 y 1973 encontraremos tan sólo 12 y 10 monografías. El año 1974 comienza ya a mostrar una ligera recuperación y ascienden ya a 13 las monografías en este año; es decir, un 1,77\% con respecto al conjunto. Este año y los siguientes, 1975 y 1976, con 13 y 16 monografías, respectivamente, empiezan a mostrar señales de recuperación en la publicación de monografías. De hecho, 1974, 1975 y 1976 van a prolo- 
gar un período de cinco años bastante fructífero, con niveles porcentuales de $1,77 \%$ y $2,18 \%$, respectivamente.

\begin{tabular}{|c|c|c|c|}
\hline Año & $N .^{\circ}$ & $\%$ & En colaboración \\
\hline 1971 & 22 & 2,99 & 2 \\
\hline 1972 & 12 & 1,63 & 3 \\
\hline 1973 & 10 & 1,36 & 1 \\
\hline 1974 & 13 & 1,77 & 0 \\
\hline 1975 & 13 & 1,77 & 1 \\
\hline 1976 & 16 & 2,18 & 2 \\
\hline 1977 & 23 & 3,13 & 3 \\
\hline 1978 & 25 & 3,39 & 0 \\
\hline 1979 & 19 & 2,58 & 1 \\
\hline 1980 & 21 & 2,85 & 0 \\
\hline 1981 & 27 & 3,67 & 1 \\
\hline 1982 & 16 & 2,18 & 0 \\
\hline 1983 & 5 & 0,68 & 0 \\
\hline 1984 & 7 & 0,95 & 9 \\
\hline 1985 & 7 & 0,95 & 0 \\
\hline 1986 & 16 & 2,18 & 0 \\
\hline 1987 & 12 & 1,63 & 3 \\
\hline 1988 & 13 & 1,77 & 2 \\
\hline 1989 & 15 & 2,04 & 3 \\
\hline 1990 & 16 & 2,18 & 3 \\
\hline 1991 & 18 & 2,43 & 6 \\
\hline 1992 & 21 & 2,86 & 2 \\
\hline 1993 & 24 & 3,27 & 5 \\
\hline 1994 & 44 & 5,98 & 6 \\
\hline 1995 & 37 & 5,02 & 9 \\
\hline 1996 & 39 & 5,28 & 7 \\
\hline 1997 & 40 & 5,44 & 11 \\
\hline 1998 & 41 & 5,57 & 2 \\
\hline 1999 & 50 & 6,79 & 16 \\
\hline 2000 & 56 & 7,60 & 12 \\
\hline 2001 & 58 & 7,88 & 4 \\
\hline Total & 736 & 100 & 114 \\
\hline
\end{tabular}

Tabla I. Producción anual de monografías y número en colaboración (1971-2001)

Así, desde los años 1977 hasta 1981 la producción anual superará casi la cifra de veinte monografías. Figuran respectivamente para estos años un volumen neto de producción anual de $23,25,19,21$ y 27 monografías. Así, los porcentajes variarán entre el año de menor producción en 1979 con un 2,58\% hasta alcanzar un porcentaje del $3,67 \%$ en 1981 . Este quinquenio tan productivo ha resultado en una producción conjunta de 115 monografías, y un porcentaje superior al $15,62 \%$ respecto a la cifra global de 736 monografías.

En general, hasta el año 1980 la producción es bastante estable, con volúmenes que van en su cota más alta de 25 monografías en 1978 hasta la más baja de 10 monografías en 1973. Sin duda, esta primera fase corresponde a la etapa de asentamiento de la publicidad en el sistema económico español y, por consiguiente, su instauración en el quehacer empresarial, especialmente gracias al desarrollo impulsor de las agencias de publicidad y del Instituto Nacional de publicidad.

Los años que siguen al productivo quinquenio anterior representan, sin duda, los niveles más bajos de producción. El año 1982 ya anuncia un descenso significativo, pues desciende la producción a un volumen de 16. Tendremos que esperar hasta el año 1986 para volver a encontrar un volumen idéntico y síntomas de recuperación. Sin duda, los años de menor producción abarcan desde 1983 hasta 1985; los volúmenes de producción de 5 y 7 monografías anuales no los volveremos a encontrar en todo el período de estudio. Estos niveles tan bajos de producción apenas representan un porcentaje por debajo del $1 \%$ anual respecto al conjunto de la producción de monografías. Esta etapa central del período de estudio, muestra así, las cifras más bajas, como señala la inflexión descendente de los años 1983, 1984 y 1985, con volúmenes de producción inferiores a las 10 monografías anuales. Como también lo fue en la actividad empresarial del sector publicitario.

A partir de 1986 el volumen de producción muestra similitudes con las cifras alcanzadas en los primeros años de estudio y anuncian en su conjunto un período más estable. Es decir, volveremos a encontrarnos con una producción de 16 monografías, equivalentes a un porcentaje del $2,18 \%$. Los años 1987 y 1988 recogen unos volúmenes de producción de 12 y 13 monografías sin llegar a alcanzar el $2 \%$ en estos años.

Los años 1989, 1990 y 1991 recogen ya sintomáticos incrementos, con volúmenes de 15,16 y 18 monografías, respectivamente. Así, van a superar anualmente porcentajes superiores al $2 \%$ en cada uno de estos años.

Continuando con el empuje de los años precedentes, los años 1992 y 1993 recogen una producción de 21 y 24 monografías, respectivamente, que vienen a representar unos porcentajes del $2,86 \%$ y $3,27 \%$ en cada caso. A partir de este momento y hasta el año final de estudio, 
encontramos un período de gran estabilidad. EI año 1994 sorprende con 44 monografías y un porcentaje casi cercano al $6 \%$ anual respecto al conjunto. Este año marca así una tendencia productiva que se extiende durante los años futuros y que configura esta etapa como la etapa de madurez de la producción monográfica española en el ámbito publicitario.

En 1995 y 1996 se produce un ligero descenso hasta las 37 y 39 monografías en cada año, que en cualquier caso distan de los descensos que habíamos visto anteriormente, ya que los porcentajes superan el $5 \%$ anual. Continuando con este signo positivo, el año 1997 señala una producción de 40 monografías, cifra similar a la del año siguiente, donde se recogen 41 monografías. Los valores porcentuales apuntan por encima del 5,40\% para estos años, 1997 y 1998. Los años finales de nuestro estudio, desde 1999 hasta el 2001, configuran ya una etapa que manifiesta la consolidación de la producción de monografías publicitarias. Encontraremos pues, volúmenes productivos de 50,56 y 58 monografías. Tan sólo en estos tres años se han producido 164 monografías que representan un porcentaje conjunto superior al $22,28 \%$.

En definitiva, es a partir de 1991 cuando se configura la etapa de consolidación de la producción científica española en el apartado de monografías. Aunque se había iniciado con cierta timidez, a partir de 1994, la producción media anual está por encima de las 37 monografías. De hecho, estos últimos años cuentan con un volumen superior a la mitad de la producción monográfica global. Tanto es así que el último año del estudio, 2001, cuenta con la producción más elevada de todo el período de estudio con una producción de 58 monografías y un porcentaje del $7,88 \%$. Claramente, este año señala la consolidación de la producción monográfica sobre temas publicitarios que anticipaban los años anteriores, y auspicia la fecundidad productiva en el nuevo siglo. Sin duda, esta última etapa pone de manifiesto la natural expansión de la publicidad como sector económico en nuestro país. Paralelamente, en el ámbito de las universidades, se produce también un fenómeno de consolidación de los estudios de Publicidad en diferentes centros académicos universitarios. El impulso académico forzosamente ha repercutido en la abundancia de publicaciones. De hecho, muchos servicios de publicaciones de diferentes universidades comienzan a editar y difundir monografías. La tendencia positiva que marca el año 2001 con el nuevo siglo rubrica el sesgo positivo de los años anteriores.

\section{Evaluación de la producción por autores}

Se elevan a cuatrocientos cuarenta y dos los autores de las 736 monografías (Anexo I). Es decir, la tasa media sería de 1.66 monografías por autor. Pues bien, de los cuatrocientos cuarenta y dos autores, son ciento cinco los autores que cuentan con más de una monografía, más del $23 \%$ respecto al conjunto de autores.

Con la excepción de un autor que cuenta con más de 20 obras monográficas, son tan sólo cinco los autores que cuentan con más de 10 monografías. Además son cuarenta y cuatro los autores que cuentan con más de 3 monografías y menos de 10. En el nivel de la producción de 2 monografías encontramos a cincuenta y cinco autores. Con una sola obra monográfica figura un grueso de trescientos treinta y siete autores.

En un nivel de análisis más pormenorizado, el autor más productivo con gran diferencia es Ferrer Roselló con 33 monografías, y acapara un porcentaje del $4,48 \%$ con respecto al conjunto de las 736 monografías. A este autor, le siguen cinco autores con un volumen de producción que media entre las 10 y las 20 obras. El autor que cuenta con mayor productividad en esta sección es Torres Padial, con 17 monografías $(2,30 \%)$. Le siguen en productividad Costa, con 16 monografías $(2,17 \%)$, y Sánchez Guzmán con 15 monografías (2,03\%). Estos autores mencionados representan una productividad superior al $2 \%$ en cada caso.

A corta distancia, figuran Sanz de la Tajada con 11 monografías y López Conejero con 10 monografías y unos porcentajes por debajo del $1,5 \%$. Con 8 monografías encontramos a un sólo autor, Basilio Gómez, con un porcentaje respecto al conjunto del $1,08 \%$. Mientras que con 7 monografías figuran Royo Vela y Santaella; porcentualmente, en cada caso, representan el $0,95 \%$. Con 6 monografías aparecen cinco autores: Barquero, Ortega, Rey Fuentes, Soler y Vallina; los porcentajes individuales apenas superan el $0,81 \%$.

Son siete los autores cuya producción individual es de 5 monografías y un porcentaje individual algo superior al 0,67\%; los mencionamos a continuación: Ferrer Rodríguez, Lema Devesa, León, Puig, Sánchez Revilla, Satué y, finalmente, Tallón. Siguiendo el nivel descendente en productividad, figuran con 4 monografías trece autores y un porcentaje, en cada caso, del $0,54 \%$. Son los siguientes: Arceo, Benavides, Eguizábal, Feijoo, Fernández Areal, García Ruescas, Martín Martín, Moliné, Pérez González, Peréz Latre, Peréz Tornero y, finalmente, 
Villafañe. Le sigue a los anteriores un grupo más numeroso de diecisiete autores con una producción individual de 3 monografías cada uno y un porcentaje individual del 0,04\%. Los anotamos: Bassat, Bigné, Calleja, Correa, Durán, González Martín, Gurrea, Heras, Martín Armario, Parra, Pérez Ruíz, Raventós, Ricarte, E. Rodríguez, Rodríguez Dieguez, Salgado y Victoria.

Finalmente, en el nivel más bajo de productividad, con tan sólo 2 monografías y un porcentaje individual del $0,27 \%$, figuran cincuenta y cinco autores. Mencionaremos sus nombres a continuación: Abril, Aguadero, Andrés, Arias, Balaguer, Baró, Beneyto, Cabal, Corredoira, Cuesta Rute, J. Echevarría, Escobar, Fernández Novoa, Ferrés, Freixas, Galí, García Fernández, García Ovejero, González Requena, González Salas, Gubern, Hernández Martínez, Herreros, López Lita, Lozano, Macía, Martínez Ramos, A. Medina, Méndiz, Morales, Moreno Menjíbar, Moreno San Marín, Noguero, Ocaña, Ochoa, Pajuelo, Piñuel, Prat, Ramallo, Ramiro, Ramos Fernández, Restrepo, Rodríguez del Bosque, Romano, Sabaté, Sáinz Fuertes, Sanabria, Sánchez Corral, Sánchez Franco, Solano, Sutil, Toro, Torres Díaz, F. Valbuena, y para concluir esta extensa relación, Xifra. Para concluir, trescientos treinta y siete autores sólo tienen una monografía en el período de estudio.

\section{Evaluación de la producción en colaboración}

Las obras en colaboración entre autores informan sobre el nivel de cooperación científica y profesional. En el apartado de la colaboración entre autores o investigadores se elevan a 114 las monografías en colaboración. Porcentualmente esta cifra representa el $15,48 \%$ respecto al conjunto de las 736 monografías.

Con respecto a la producción general de monografías en colaboración, la evolución en general es bastante estable y con una evolución positiva a lo largo del período de estudio (Tabla I). De hecho, hasta la década de 1990 la producción se sitúa en unos volúmenes de 1, 2 ó 3 monografías, o es nula. Destaca la elevada productividad del año 1984 con 9 monografías. Curiosamente los dos años precedentes y los dos posteriores carecen de producción de monografías en colaboración. Sin embargo, a partir de 1990 la producción tiene un saldo positivo ascendente y continuo. Tanto es así que vamos a encontrar un volumen de 11 monografías en 1997, de 12 monografías en el 2000, y de 16 monografías en 1999, siendo este el año con la mayor productividad. Más de la mitad de la pro- ducción de las monografías en colaboración tiene lugar a partir de la década de 1990.

Las 114 monografías en colaboración son obra de noventa y siete autores. Es decir, el 21,89\% del conjunto de los cuatrocientos cuarenta y dos autores, lo son de obras en colaboración. Además, debemos resaltar que de estos noventa y siete autores, treinta de ellos han publicado además otras monografías, bien en solitario o en colaboración.

Entre los autores con mayor productividad en las monografías en colaboración, destacan cinco autores con una producción individual de 3 monografías y un porcentaje del $2,63 \%$ respecto al conjunto de las monografías en colaboración. Nos referimos a los siguientes autores: Bigné, Fernández Areal, Ferrer Roselló, Lema y Rey.

En un nivel más bajo de productividad, figuran siete autores con 2 monografías en colaboración. Es decir, representan un porcentaje individual del $1,75 \%$. Los mencionamos a continuación: J. L. Arceo, Benavides, Pérez González, Ramallo, Rodríguez del Bosque, Royo y Soler.

Finalmente, figura un grupo de ochenta y cinco autores con una sola monografía en colaboración, y un porcentaje del $0,87 \%$ en cada caso. Es interesante destacar en este grupo de autores con una sola monografía en colaboración, que dieciocho autores cuentan además con monografías en solitario. Nos referimos a Barquero, Correa, Costa, Eguizábal, González Requena, Heras, Hernández Martínez, Martín Armario, Méndiz, Ortega, Piñuel, Restrepo, J. L. Rodríguez, Sánchez Franco, Sánchez Guzmán, Sanz de la Tajada, Toro y Villafañe.

\section{Evaluación de la producción institucional}

En el análisis dedicado a la producción institucional hemos encontrado problemas a la hora de buscar la vinculación institucional o profesional de algunos autores, especialmente entre aquellos no vinculados a universidades, dada la gran movilidad profesional frecuente entre los profesionales dedicados a la actividad publicitaria. En muchos casos, sin embargo, algunos de los profesionales son también docentes. En estos casos, dada la necesidad de elección, hemos optado por la vinculación universitaria. Cuando se ha dado la particularidad de varias instituciones, hemos optado por la vigente en el momento de la publicación de la monografía.

Tampoco han ayudado las omisiones de los mismos editores. Nos hemos encontrado con muchas monografías donde sólo figura la inicial para el nombre y tan sólo un apellido; mientras 
que en otra monografía del mismo autor figuraban el nombre completo y el segundo apellido.

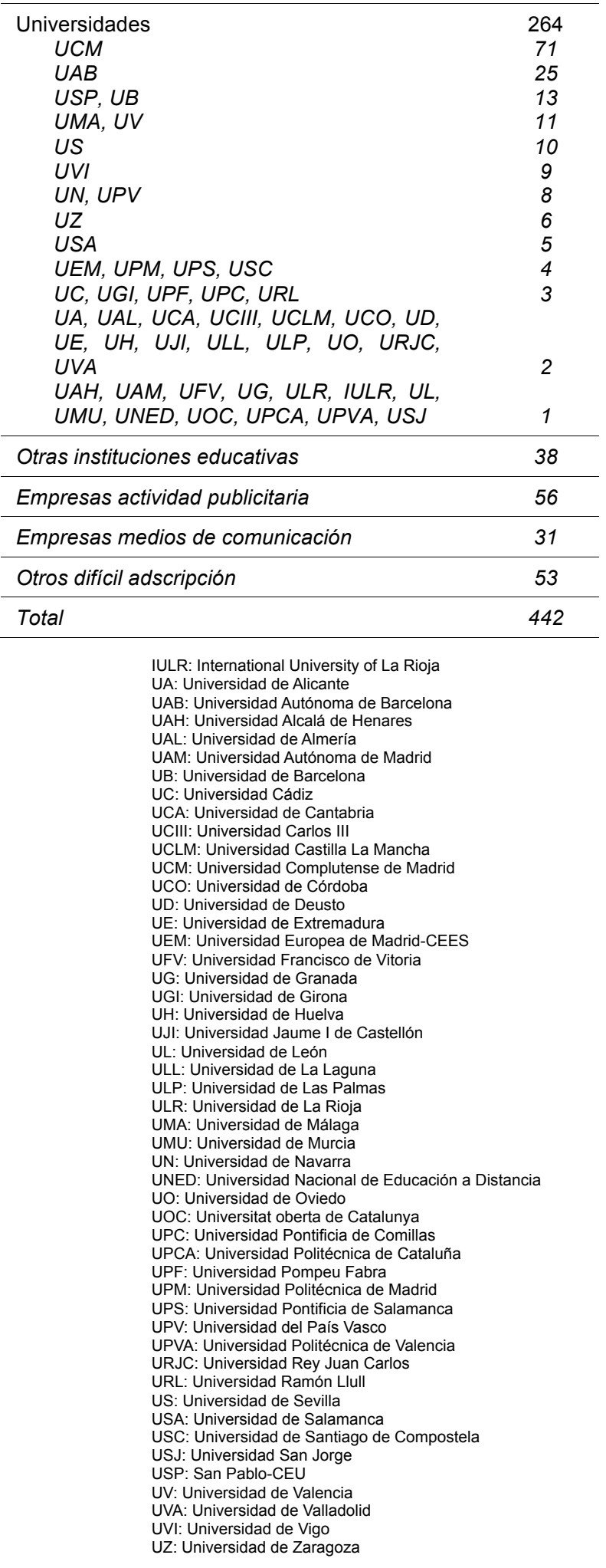

Tabla II. Vinculación institucional de los autores de las monografías
En muchas ocasiones tampoco se hace referencia a la profesión clara del autor y se le menciona como "profesor", "investigador", "publicitario", "publicista", etc. Salvados la mayoría de los problemas, hemos conseguido encontrar la adscripción institucional o universitaria para la mayor parte de los autores. En ocasiones, hemos solventado el problema poniéndonos en contacto con ciertas instituciones y empresas.

Los cuatrocientos cuarenta y dos autores están vinculados en su gran parte a la docencia universitaria y educativa, al ámbito profesional de la actividad publicitaria y, por proximidad a este último, al sector de los medios de comunicación (Tabla II). Iniciaremos nuestro análisis con las instituciones que cuentan con un mayor número de autores o investigadores vinculados a ellas, es decir, por las más productivas. Así, el número de autores vinculados a alguna institución universitaria asciende a doscientos sesenta y cuatro autores, distribuidos entre cuarenta y nueve universidades. En su conjunto, los autores vinculados como titulares o asociados de universidad representan más del $59 \%$ respecto al conjunto global.

La Universidad Complutense de Madrid se sitúa a la cabeza con setenta y un autores vinculados a ella, que representan un porcentaje superior al 16\%. A bastante distancia, la Universitat Autónoma de Barcelona aporta veinticinco autores $(5,6 \%)$. La Universitat de Barcelona y la Universidad San Pablo-CEU aportan cada una trece autores $(2,94 \%)$. La Universidad de Málaga y la Universidad de Valencia aportan, respectivamente, once autores (2,48\%). La Universidad de Sevilla contribuye con diez autores (2,26\%), mientras que la Universidad de Vigo cuenta con nueve autores $(2,03 \%)$. La Universidad de $\mathrm{Na}$ varra y la Universidad del País Vasco aportan cada una ocho autores (1,80\%). La Universidad de Zaragoza contribuye con seis autores $(1,35 \%)$, mientras que la Universidad de Salamanca contribuye con cinco $(1,13 \%)$. Cuatro universidades aportan cuatro autores cada de ellas $(0,90 \%)$. Se trata de la Universidad Europea de Madrid CEES, la Universidad Politécnica de Madrid, la Universidad Pontificia de Salamanca y la Universidad de Santiago de Compostela. Respecto al conjunto de autores adscritos a universidades aportan un porcentaje del $1,50 \%$ cada una de ellas.

En un nivel más bajo de productividad de las universidades, se sitúan cinco universidades que aportan tres autores cada una de ellas $(0,67 \%)$. Son la Universidad de Cádiz, la Universidad de Girona, la Universitat Pompeu Fabra, la Universidad Pontificia de Comillas y la Universidad Ramón Llull. Siguiendo el orden 
descendente, quince universidades contribuyen con dos autores cada una $(0,45 \%)$; son la Universidad de Alicante, Universidad de Almería, Universidad de Cantabria, Universidad Carlos III de Madrid, Universidad de Castilla La Mancha, Universidad de Córdoba, Universidad de Deusto, Universidad de Extremadura, Universidad de Huelva, Universitat Jaume I, Universidad Rey Juan Carlos, Universidad de La Laguna, Universidad Las Palmas de Gran Canaria, Universidad de Oviedo y la Universidad de Valladolid.

Finalmente, en el nivel más bajo de productividad de las universidades, figuran trece universidades que aportan un sólo autor cada una de ellas $(0,22 \%)$; son la de Alcalá de Henares, Autónoma de Madrid, Francisco de Vitoria, de Granada, de La Rioja, International University of La Rioja, de León, de Murcia, Nacional de Educación a Distancia, Universitat Oberta de Catalunya, Politécnica de Catalunya, Politécnica de Valencia y San Jorge de Zaragoza.

También es importante destacar treinta y ocho autores $(8,59 \%)$ distribuidos entre diferentes centros, escuelas e instituciones con una proyección hacia la enseñanza no universitaria.

Por su parte, un grueso de cincuenta y seis autores $(12,66 \%)$ está vinculado claramente con alguna actividad o empresa específica del mundo profesional publicitario, sin que exista concentración importante en alguna empresa determinada.

Próximos a los anteriores, destaquemos a treinta y un autores $(7,01 \%)$ relacionados claramente a alguna actividad específica centrada en los medios de comunicación, siendo la mayor parte periodistas especializados en publicidad. Finalmente, figuran dispersos cincuenta y tres autores $(11,99 \%)$ en muy diferentes profesiones, como políticos o magistrados, entre otras.

\section{Evaluación de la producción por idiomas}

El castellano es la lengua en que se han escrito la mayor parte de las monografías, que ascienden a 704, que vienen a representar un porcentaje superior al $95 \%$ con respecto al conjunto de las 736 monografías. El número de monografías escritas en lenguas diferentes al castellano asciende a 32 , lo que significaría un porcentaje superior al $4 \%$ respecto al conjunto de las 736 monografías. Le sigue al castellano como lengua más activa, el catalán, representado por 21 monografías. La primera monografía escrita en esta lengua aparece en 1983 y está firmada por Siurana, aunque el empuje mayor se sitúa a partir del año 1992. Las monografías escritas en gallego ascienden a 9, es decir, apenas un $1,22 \%$ con respecto al conjunto. La primera obra en gallego la encontramos en el año 1993, firmada por Cermeño. También la lengua inglesa ha merecido el interés de dos monografías publicadas en 1996 y 1997.

\section{Conclusiones}

1. La producción global de monografías se eleva a 736. Los años de mayor producción corresponden a la etapa que se inicia en 1994 hasta el final del período de estudio, cuando se produce un incremento de la producción como se advierte en las 365 monografías que representan un porcentaje cercano al $49 \%$. También en el año 2001 se alcanza la máxima producción con 58 monografías y un porcentaje del $7 \%$.

En general la etapa de menor producción corresponde a la que abarca desde 1981 hasta 1990. El año 2001 manifiesta la tendencia al alza en la producción de monografías.

2. Las 736 monografías han sido realizadas por cuatrocientos cuarenta y dos autores. El autor más productivo es Ferrer Roselló con 33 monografías $(4,48 \%)$. A continuación, en orden descendente en productividad, figuran Torres Padial con 17 monografías (2,30\%), Costa Solá con 16 $(2,17 \%)$ y Sánchez Guzmán con 15 (2,03\%). Ciento cinco autores tienen más de una monografía $(23 \%)$ frente a trescientos treinta y siete autores con una sola monografía $(45,78 \%)$.

3. La producción global de monografías en colaboración se eleva a 114, que representan un porcentaje del $15,48 \%$. La evolución de la producción en general es positiva y estable a lo largo del período de estudio. Aunque ya existen monografías en colaboración desde los primeros años del período de estudio, casi la mitad de estas monografías en colaboración han sido realizadas a partir de 1991, y ascienden a 76 monografías hasta el año 2000. Estas 114 monografías en colaboración han sido realizadas por noventa y siete autores, es decir, el $21 \%$ con respecto al conjunto global de cuatrocientos cuarenta y dos autores. Entre los autores más productivos de monografías en colaboración, destacan Bigné, Fernández Areal, Ferrer Roselló, Lema Devesa y Rey, quienes cuentan cada uno con tres monografías en colaboración.

4. Los niveles de producción referentes a adscripción a universidades e instituciones muestran que la mayor productividad corresponde a las a universidades con doscientos sesenta y cuatro autores, distribuidos entre cuarenta y nueve universidades. Los autores vinculados como titulares o asociados de universidad re- 
presentan más del $59 \%$ respecto al conjunto global. Entre las universidades con mayor número de autores vinculados destaca la Universidad Complutense de Madrid con setenta y un autores (16\%). A distancia figura la Universitat Autónoma de Barcelona con veinticinco $(5,6 \%)$. Con trece autores $(2,94 \%)$ figuran la Fundación Universitaria San Pablo-CEU y la Universidad de Barcelona. La Universidad de Málaga y la Universidad de Valencia aportan once autores $(2,48 \%)$ cada una. Finalmente la Universidad de Sevilla $(2,26 \%)$ contribuye con diez autores. Además, destacan treinta y ocho autores $(8,59 \%)$ distribuidos entre diferentes centros, escuelas e instituciones no universitarias.

Dentro de la actividad publicitaria profesional destacan cincuenta y seis autores $(12,66 \%)$ vinculados a alguna empresa del sector publicitario. Por proximidad, también debemos anotar a treinta y un autores $(7,01 \%)$ vinculados a los medios de comunicación. Finalmente, existen cincuenta y tres autores $(11,99 \%)$ dispersos en muy diferentes profesiones.

5. La lengua predominante es el castellano con 704 monografías, cifra que representa un porcentaje superior al 95\%. En catalán figuran 21 monografías $(2,85 \%)$; en lengua gallega, 9 monografías ( $1 \%)$; y dos en inglés.

\section{Referencias}

Delgado López-Cózar, E.; Torres Salinas, D.; Jiménez Contreras, E.; Ruíz Pérez, R. (2006). Análisis bibliométrico y de redes sociales aplicado a las tesis bibliométricas defendidas en España (1976-2002): Temas, escuelas científicas y redes académicas. // Revista Española de Documentación Científica. 29: 4 (2006) 493-524.

Finkenstaedt, T. (1990). Measuring research performance in the Humanities. // Scientometrics. 19 (1990) 409-417.

García Ruescas, Francisco (2000). Historia de la publicidad y del arte comercial en España. Madrid: Editorial Arus, 2000.

Glänzel, Wolfgang (2003). Bibliometrics As a Research Field: A Course on Theory and Application of Bibliometric Indicators. Course Handouts. Leuven: Katholieke Universiteit Leuven, 2003.

Hopkins, Claude C. (1980). Publicidad científica. Madrid: Eresma Edic, 2003. (Trad. M.A. González Lob)

Jones, Daniel E. (1998). Investigación sobre comunicación en España. Evolución y perspectivas. // Zer, 5 (1998) 13-51.

Jones, Daniel E.; Baró I Queralt, Jaume (1997a). Tesis doctorals i traballs de recerca universitaris sobre comunicació als països Catalans, 1954-1996: Aproximació bibliomètrica. // Anàlisis. 20 (1997) 157-187.

Jones, Daniel E. (1997b). Investigación sobre comunicación social en la España de las autonomías. // Anàlisi. 21 (1997) 101-120.

López López, Pedro (1996a). La investigación bibliométrica en España (tesis doctorales). // Revista Española de Documentación Científica. 19: 1 (1996) 84-89.
López López, Pedro (1996b). Introducción a la bibliometría. Valencia: Editorial Promolibro, 1996.

López Piñero, J.M.; Terrada, M.L. (1992a). Los indicadores bibliométricos y la evaluación de la actividad médicocientífica. (I) Uso y abusos de la bibliometría. // Medicina Clínica. 98 (1992) 64-68.

López Piñero, J.M.; Terrada, M.L. (1992b). Los indicadores bibliométricos y la evaluación de la actividad médicocientífica. (II) La comunicación científica en las distintas áreas de las ciencias sociales. // Medicina Clínica. 98 (1992) 101-106.

López Piñero, J.M.; Terrada, M.L. (1992c). Los indicadores bibliométricos y la evaluación de la actividad médicocientífica. (III) Los indicadores de producción, circulación y dispersión, consumo de información y repercusión. // Medicina Clínica. 98 (1992) 142-148.

López Piñero, J.M.; Terrada, M.L. (1992d). Los indicadores bibliométricos y la evaluación de la actividad médicocientífica. (IV) La aplicación de los indicadores. // Medicina Clínica. 98 (1992) 384-388.

López Yepes, José (1981) La organización del Centro de documentación en la agencia de publicidad. // López Yepes, José (Ed). Estudios de Documentación general e informativa. Madrid: Seminario "Millares Carlo", Universidad Nacional de Educación a Distancia, Centro Regional Las Palmas, 1981. 413-418.

Marcos Recio, J.C.; Sánchez Vigil, J.M.; Olivera Zaldua, M. (2010): Modelos de gestión documental en las agencias de publicidad. // El Profesional de la Información. 19 (2) (2010) 175-183.

Marcos Recio, J.C. (2003): Estrategias documentales en agencia de publicidad. // BiD: Textos universitaris de biblioteconomia i documentació. 11 (2003).

Maroto, Ricardo; Martín Oviedo, J.M.; Granda, I.; Feo, J.; Retuerto, E.; Rabassa, B.; Tulla, A.; Plana. E.; Lozano, D.; Moline, M.: Avellaneda, M.T.; Anguera, F.; Borrell, J.; Equipo Revista Control (1971). Sistematización básica del conocimiento publicitario. Madrid: Revista de Control de Publicidad y Ventas, 1971.

Martín Martín, Fernando (1983). Las bases de datos publicitarias: Estudio bibliográfico de la comunicación publicitaria española (1900-1983). Madrid: Duplo, S.A. y Departamento de Documentación de la Facultad de Ciencias de la Información, Universidad Complutense de Madrid, 1983.

Martínez Pestaña, María Jesús (2004). La producción de tesis doctorales sobre temas publicitarios (1971-2001). // Documentación de las Ciencias de la Información. 27 (2004) 237-267.

Méndiz Noguero, Alfonso (2000). Orígenes, evolución y desafíos actuales de la docencia publicitaria en España. // Comunicación y Sociedad. XIII: 2 (2000) 181-225.

Nederhof, A.J.; Van Raan, R.A.; De Bruin, R.E.; Dekker, P.J. (1989). Assessing the usefulness of bibliometric indicators for the humanities and the social behavioral sciences: A comparative study. // Scientometrics. 15 (1989) 423-435.

Ortega Martínez, Enrique (1997). Comunicación publicitaria. Madrid: Ediciones Pirámide, S.A, 1997.

Osareh, Farideh (2003). The use and application of multivariate analysis techniques in bibliometric and scientometric studies. // Iranian Journal of Information Science \& Technology. 1: 2 (2003) 59-71.

Palomar, T.; García-Heras, M.; Villegas, M.A. (2009). Archaelogical and historical glasses: A bibliometric study. // Boletín de la Sociedad Española de Cerámica y Vidrio. 48:4 (2009) 187-194.

Perez Gonzalez, Rafael Alberto (2000). La docencia en publicidad. // Heras Pedrosa, Carlos de las (Coord.). La 
década de oro de la publicidad en España: años 60. Málaga: Ayuntamiento de Málaga. (2000). 39-58.

Prat Gaballí, Pedro (1917). Una nueva técnica, la publicidad científica. Barcelona: Edit. Cámara de Comercio y Navegación, 1917.

Prat Gaballí, Pedro (1920). Los caminos científicos de la publicidad. Barcelona: Edit. Cultura, 1920.

Ramiro Castellblanque, Mariano (2001). Estructura de la actividad publicitaria: La industria de la publicidad de la A a la Z, España: una caso extrapolable. Ediciones Paidós Ibérica, S.A, 2001.

Sánchez Guzmán, José Ramón (1980). El tratamiento científico de la publicidad. // Publitecnia. 54 (1980) 125-143.

Sánchez Guzmán, José Ramón; Anleo, J.G. (1979). Algunos aspectos de la publicidad en España. Madrid: Instituto Nacional del Consumo, 1979

Sánchez Revilla, Miguel Ángel (1998). La inversión publicitaria en los medios: De los grandes crecimientos de la transición y la década de los 80 , a la crisis y madurez de finales del siglo. // Ip Mark. 511 (1998) 19-22.
Van Leeuwen, Thed (2006). The application of bibliometric analyses in the evaluation of social science research. Who benefits from it, and why it is still feasible. // Scientometrics. 66: 1 (2006) 133-154.

Villafañe Gallego, Justo (2001). El estado de la publicidad en España, informe 2001. Madrid: Ediciones Pirámide, S.A, 2001.

Villagrá Rubio, A. (1992). Scientific production of Spanish universities in the fields of social sciences and language. // Scientometrics. 24: 1 (1992) 3-19.

Zulueta, María Ángeles (2002). Bibliometría y métodos bibliométricos // López Yepes, J. (Coord.). Manual de Ciencias de la Documentación. Madrid: Ediciones Pirámide, 2002. 117-136.

Zulueta, María Ángeles; Cabrero, A.; Bordons, M. (1999). "Identificación y estudio de grupos de investigación a través de indicadores bibliométricos. // Revista Española de Documentación Científica. 22 (1999) 333-347.

\section{Anexo I. Relación de autores de monografías y número de monografías por autor}

Abril, G. (2)

Agote, F.J. (1)

Aguadero F, F (2)

Aguilar Gutiérrez, J.A. (1)

Aguilar Piñal, F. (1)

Aguilera Gamoneda, J. (1)

Aguilera Moyano, M. (1)

Aguilera Perelló, O. (1)

Aguilera Podadera, J. (1)

Aladro Vico, E. (1)

Albisu, L.M. (1)

Alcaraz Varo, E. (1)

Alfonso Durán, J. (1)

Alonso Alonso, J.M. (1)

Alonso Erausquín, M. (1)

Altares, P. (1)

Alva de Diego, V. (1)

Álvarez Domínguez, T. (1)

Álvarez, J.T. (1)

Amorós i Pons, A. (1)

Andrés, A.J. (2)

Angeles Villena, J. (1)

Antona Illanes, A. (1)

Aragay Prades, J.J. (1)

Aragón Varo, A. (1)

Aragonés Correderas, P. (1)

Arce, R. (1)

Arceo Vacas, A. (1)

Arceo Vacas, J.L. (4)

Arias (Ruiz), A. (2)

Arquillos Toribio, F.E. (1)

Arregui Sierra, M.A. (1)

Ávila Sánchez, M.A. (1)

Aznar Gómez, H. (1)

Balaguer, M.L. (2)

Bando Casado, H.C. (1)

Baños (González), M. (1)

Barcelo (Valls), C. (1)

Baró i Balbé, M.J. (2)

Barona Vilar, S. (1)

Barquero Cabrero, J.D. (6)

Barranco Sáiz, F.J. (1)

Barrio García, S. (1)

Basilio Gómez, J. (8)

Bassat Coen, L. (3)
Bassets, L. (1)

Batista González, M.P. (1)

Becerra Castillo, A. (1)

Beerli Palacio, A. (1)

Benavides Delgado, J. (4)

Beneyto, J. (2)

Benito del Valle, A. (1)

Berger Ibarra, F. (1)

Bermejo, A. (1)

Biedma López, J. (1)

Bigné Alcañiz, J.E. (3)

Blanco Rodríguez, L. (1)

Blázquez Fernández, N. (1)

Bocigas, O. (1)

Bonet (Albero), E. (1)

Bonsiepe, G. (1)

Borja Sole, L. (1)

Borrini, A. (1)

Brisset Martí, D. (1)

Briz Escribano, J. (1)

Brugada, M. (1)

Bueno García, A. (1)

Burgos, D. (1)

Burriel Álvarez, J.M. (1)

Bustamante Ramírez, E. (1)

Cabal González, M. (2)

Cabrera, J.A. (1)

Calleja, J.L. (3)

Calvo Fernández, S. (1)

Calvo Serraller, F. (1)

Campo, M. (1)

Campos López, C. (1)

Camps Monsech, J.R. (1)

Capriotti, V.P. (1)

Cardona, D. (1)

Caro, A. (1)

Carrasco Andrino, M.M. (1)

Casado Juan, F. (1)

Casanova Seuema, L. (1)

Casanovas Cristóbal, J. (1)

Casero, J.M. (1)

Castillo Cuervo, F. (1)

Celma Querol, J. (1)

Cerezo Arriaza, M. (1)

Cermeño, J. (1)
Chaves, N. (1)

Cirigliano, G.F.J. (1)

Clemente (Díaz), M. (1)

Coll Vinent, R. (1)

Conde, F. (1)

Conejero López, M. (10)

Corazón, A. (1)

Correa García, R.I. (3)

Corredoira y Alfonso, L. (2)

Cortés de los Ríos, M.E. (1)

Costa Solá-Segalés, J. (16)

Cruz Samper, C. (1)

Cuesta Cambra, U. (1)

Cuesta Rute, J.M. (2)

Cuesta, U. (1)

Díaz Arias, R. (1)

Díaz Esarte, X. (1)

Díaz Pérez, A. (1)

Díaz Santana, H. (1)

Díez Arroyo, M. (1)

Díez de Castro, E. (1)

Díez Medrano, J. (1)

Durán (Pitch), J.A. (3)

Duro Moreno, M. (1)

Echevarría Vicente, M.A. (1)

Echevarría, J. (2)

Eguizábal Maza, R. (4)

Elías Monclus, J. (1)

Enebral Casares, F.J. (1)

Escobar de la Serna, L. (2)

Espadalé Vergés, B. (1)

Espinosa Calabuig, R. (1)

Espinosa, M.P. (1)

Esteve (Zarazaga), J.M. (1)

Expósito, M. (1)

Faura, f. (1)

Feijoo, E. (4)

Fernández Areal, M. (4)

Fernández Cuesta, N. (1)

Fernández Garrido, J. (1)

Fernández Novoa, C. (2)

Fernández Pellitero, M. (1)

Fernández Sanz, J.M. (1)

Fernández Shaw, F. (1)

Ferraz Martínez, A. (1)
Ferré Trenzano, J.M. (1)

Ferreiro Currás, F. (1)

Ferrer Forns, A. (1)

Ferrer Márquez, M.J. (1)

Ferrer Multiva, I. (1)

Ferrer Rodríguez, E. (5)

Ferrer Roselló, C. (33)

Ferrero, J.J. (1)

Ferrés i Prats, J. (2)

Fesser, E. (1)

Fita Trías, J. (1)

Fleming, P. (1)

Font Blanch, D. (1)

Fontcuberta Vernet, J. (1)

Francoy, M. (1)

Fratini, E. (1)

Freixas Aranguren, E. (2)

Furones Ferré, M.A. (1)

Galí, J.M. (2)

Galván Morera, J.F. (1)

Garay Martín, T. (1)

García Ballesteros, J. (1)

García de Polavieja, J.C. (1)

García Echevarría, S. (1)

García Fernández, N. (2)

García Filgueira, M. (1)

García García, I. (1)

García González, A. (1)

García Guatas, M. (1)

García Herrero, G. (1)

García Jiménez, J. (1)

García López, M. (1)

García Matilla, E. (1)

García Ovejero, J. (2)

Garcia Ruescas, F. (4)

García Uceda, M. (1)

García Vélez, D. (1)

García-Lavernia, J. (1)

Garriga i Puig, J. (1)

Gibert Rahola, J. (1)

Gil Albarova, A. (1)

Gil Pizarro, J. (1)

Gil, R. (1)

Gladish Butt, C.P. (1)

Gómez Díaz, A. (1) 
Gómez Jiménez, M.A. (1) Goméz-Reino, E. (1) Gomis Sanahuja, L. (1) González Ballesteros, T. (1) González García, F.A. (1) González Lacita, P. (1) González Lobo, M.A. (1) González Martín, J.A. (3) González Requena, J. (2) González Salas, A. (2) González-Anleo, J. (1) González, J.L. (1)

Gorostiaga, E. (1) Gorris Torres, J.M. (1)

Goyanes, E. (1)

Granjel, L. (1)

Gubern, R. (2)

Guerra Tapia, A. (1)

Gurrea Saavedra, A. (3)

Gutiérrez Espada, L. (1)

Gutiérrez Ordóñez, S. (1)

H. de la mota, I. (1)

Heras Pedrosa, C. (3)

Hernández Martínez, C. (2)

Hernando Cuadrado, L.A. (1)

Herreros Arconada, M. (2)

Higueras Vicente, I. (1)

Hojas Hojas, L.I. (1)

Homedes Clavero, J. (1)

Hormigo Hernández, E.C. (1)

Huici Módenes, A. (1)

Igartua Perosanz, J. (1)

Iglesias González, F. (1)

Iniesta García, S. (1)

Izquierdo Navarro, F. (1)

Jordán, A. (1)

Jordana, J.V. (1)

Jordi González, R. (1)

Jurado Gómez, E. (1)

Lavilla Raso, M. (1)

Leal López, F.J. (1)

Lema Devesa, C. (5)

León Saéz, J.L. (5)

Liria, E. (1)

Llovet, J. (1)

Lluch Picazo, C. (1)

Lomas, C. (1)

López Eire, A. (1)

López Franco, J. (1)

López García, M.J. (1)

López Gómez, A. (1)

López Guerra, L. (1)

López Lita, R. (2)

López, E. (1)

Lorente García, J. (1)

Lozano Domínguez, F. (2)

Lucas, Antonio (1)

Luxán Meléndez, S. (1)

Macía Mercadé, J. (2)

Madrenas i Boadas, C. (1)

Maldonado T. (1)

Manera Bassa, J. (1)

Mangino, L. (1)

Mañas Gómez, C. (1)

Mapelli Cafarena, B. (1)

Marce i Puig, F. (1)

Marchamalo, J. (1)

Marcos González, T. (1)

Marín, C. (1)

Maroto, R. (1)

Marqués Romeral, E. (1)

Martí Escayol, M.A. (1)
Marti, S. (1)

Martín Armario, E. (3)

Martín de Antonio, R. (1)

Martín Martín, F. (4)

Martín Oviedo, J. (1)

Martín Poyo, I. (1)

Martín Serrano, M. (1)

Martín y Pérez de Nanclares, J. (1)

Martín, L. (1)

Martínez y Martínez, M. (1)

Martínez López, R. (1)

Martínez Ramos, E. (2)

Martínez, Q. (1)

Masso Tarruella, R. (1)

Mata Grande, C. (1)

Mata, F. (1)

Mazo del Castillo, J.M. (1)

Medina Laverón, M. (1)

Medina, A. (2)

Méndez Tomás, R.M. (1)

Méndez Garrido, J.M. (1)

Méndiz Noguero, A. (2)

Merino Rivera, M.D. (1)

Mínguez Vela, A. (1)

Miquel Peris, S. (1)

Mirandes i Grabolosa, N. (1)

Mola, I. (1)

Molero Ayala, V.M. (1)

Molero García, M. (1)

Moliné Golovart, M. (4)

Moliner, J.T. (1)

Moll de Miguel, J. (1)

Monfort, M. (1)

Montoya Vilar, N. (1)

Moragas Spa, M. (1)

Morales Navarro, C. (2)

Moreno Martín, A. (1)

Moreno Menjíbar, J.L. (2)

Moreno San Martín, J. (2)

Moreno y Bravo, E. (1)

Moriyón Mojica, C. (1)

Mota Oreja, J. (1)

Mourelle de Lema, M. (1)

Muela Molina, C. (1)

Muntanyà i Puig, T. (1)

Muñagorri Laguía, I. (1)

Muñoz, J.J. (1)

Musitu, G. (1)

Nacher, E. (1)

Navarro Herranz, M. (1)

Navarro Valls, J. (1)

Nebot, J.E. (1)

Nó, J. (1)

Noguera, J. (1)

Noguero i Grau, A. (2)

Núñez de Prado, S. (1)

Nuñez Ladeveze, L. (1)

Núñez, P. (1)

Ocaña, F. (2)

Ochoa Santamaría, I. (2)

Oejo Montano, E. (1)

Olamendi, G. (1)

Orive, P. (1)

Ortega Martínez, E. (6)

Ortiz, I. (1)

Otero Alvarado, M.T. (1)

Pacheco Rueda, M. (1)

Pajuelo de Arcos, C. (2)

Parra, E. (3)

Parramón, J.M. (1)

Pascual, J.M. (1)
Peña Marín, C. (1)

Perales Castro, A. (1)

Pérez Gauli, J.C. (1)

Pérez González, R.A. (4)

Pérez Latre, F.J. (4)

Pérez Ruíz, M.A. (3)

Pérez Tornero, J.M. (4)

Pérez Villar, J. (1)

Pescador Martín, M. (1)

Pinazo, D. (1)

Pinillos Suárez, P.J. (1)

Pino Abad, M. (1)

Pino, A. (1)

Piñuel Raigada, J.L. (2)

Piserra Tello, J. (1)

Pitarch, A.J. (1)

Plans Álvarez, J. (1)

Porras, C. (1)

Porta Jacques, F. (1)

Prat Gaballí, P. (2)

Puig de la Bellacasa, R. (1)

Puig, J.J: (5)

Quinteiro Noya, F. (1)

Ramallo, F.F. (2)

Ramiro Castellblanque, M. (2)

Ramos Fernández, F. (2)

Ramos Serrano, M. (1)

Raventós Rabinat, J.M. (3)

Rebolledo San Martín, M.A. (1)

Reinares Lara, P. (1)

Renau, J. (1)

Restrepo Pardo, E. (2)

Rey Fuentes, J. (6)

Reyzábal Rodríguez, M.V. (1)

Ricarte Bescós, J.M. (3)

Rivero Sánchez, J.A. (1)

Rivillas Jurado, J. (1)

Rodergas i Payés, R. (1)

Rodríguez Méndez, J.M. (1)

Rodríguez Vilanova, E. (3)

Rodríguez del Bosque, I.A. (2)

Rodríguez Diéguez, J.L. (3)

Rodríguez Pardo, J. (1)

Rodríguez Pedremonte, A. (1)

Rodríguez Sánchez, C. (1)

Roig, M. (1)

Rojo Villada, P.A. (1)

Román Portas, M. (1)

Romano, V. (2)

Romero Rubio, A. (1)

Royo Vela, M. (7)

Ruíz Collantes, X. (1)

Ruíz Ogarrio, F. (1)

Sabaté i López, J. (2)

Saborit Viguer, J. (1)

Sáinz Fuertes, A. (2)

Salas Nestares, M.I. (1)

Salazar Bello, J. (1)

Salgado Carrión, J.A. (3)

Sanabria, F. (2)

Sánchez Ventura, J.M. (1)

Sánchez Corral, L. (2)

Sánchez de Muniain, J.M. (1)

Sánchez Franco, M.J. (2)

Sánchez Grajel, L. (1)

Sánchez Guzmán, J.R. (15)

Sánchez López, R. (1)

Sánchez Revilla, M.A. (5)

Santaella López, M. (7)

Sanz de la Tajada, L.A. (11)

Sanz Díaz, A. (1)

Sanz Rubiales, J. (1)
Satué Llop, E. (5)

Saz rubio, M. (1)

Senabre Francés, J. (1)

Sintes Pelaz, R. (1)

Siurana, V. (1)

Sobrino Manzanares, M.L. (1)

Solana, D. (1)

Solanas Donoso, J. (1)

Solano Fleta, L. (2)

Soler Pujals, P. (6)

Soria, C. (1)

Soriano Soriano, C. (1)

Suárez Alonso, M. (1)

Summers, G. (1)

Sutil Martín, D.L. (2)

Tallón García, J. (5)

Tato Plaza, A. (1)

Tavares de Carbalho, L.F. (1)

Todoli, J. (1)

Toro Martín, J.M. (2)

Torre Navarro, R. (1)

Torrent, A.M. (1)

Torres Díaz, F. (2)

Torres Padial, R. (17)

Trece, B. (1)

Tubau, I. (1)

Ullod Pujol, A.M. (1)

Unsain Azpiroz, J.M. (1)

Urrutia, J. (1)

Urzaiz, J. (1)

Valencia López, V. (1)

Vales Iglesias, A. (1)

Vallina Velilla, M. (6)

Valls, J.F. (1)

Valvuena, F. (2)

Vaquera, C. (1)

Vázquez Montalbán, M. (1)

Vázquez, I. (1)

Velo Velo, M. (1)

Ventura, L. (1)

Victoria Más, J.S. (3)

Vidal Silva, P. (1)

Villafañe Gallego, J. (4)

Vinyes-M. i Gassó, T. (1)

Xifra Triadú, J. (2)

Yaguez, R.A. (1)

Zunzunegui Díez, S. (1) 